\title{
Estimating the global risk of anthropogenic climate change
}

\author{
Alexandre K. Magnan $\odot 1,2 \llbracket$, Hans-Otto Pörtner $\oplus^{3}$, Virginie K. E. Duvat $\oplus^{2}$, Matthias Garschagen $\oplus^{4}$, \\ Valeria A. Guinder ${ }^{5}$, Zinta Zommers ${ }^{6,10}$, Ove Hoegh-Guldberg ${ }^{7}{ }^{7}$ and Jean-Pierre Gattuso ${ }^{11,8,9}$
}

The three recent Special Reports of the IPCC provide an opportunity to understand overarching climate risk, as they cover a wide diversity of risks to natural and human systems. Here we develop a scoring system to translate qualitative IPCC risk assessments into risk scores that, when aggregated, describe global risk from climate change. By the end of this century, global climate risk will increase substantially with greenhouse gas emissions compared to today (composite risk score increase of two- and fourfold under RCP2.6 and RCP8.5, respectively). Comparison of risk levels under $+1.5^{\circ} \mathrm{C}$ and $+2{ }^{\circ} \mathrm{C}$ suggests that every additional $0.5^{\circ} \mathrm{C}$ of global warming will contribute to higher risk globally (by about a third). Societal adaptation has the potential to decrease global climate risk substantially (by about half) under all RCPs, but cannot fully prevent residual risks from increasing (by one-third under RCP2.6 and doubling under RCP8.5, compared to today).

Sentin everal frameworks have been developed ${ }^{1-6}$ to understand the increasing risks of socio-ecosystem degradation and collapse from the human-driven disturbances of the last few centuries to the terrestrial, marine and climate systems. Here we build on the expert judgement-based assessment by the Intergovernmental Panel on Climate Change (IPCC) $)^{5,6}$ to estimate the composite risk from anthropogenic climate change by the end of the 21st century, hereafter 'global climate risk', defined as the globally aggregated climate change-related risks to terrestrial and ocean systems across ecological and human dimensions at all latitudes and levels of socio-economic development. Such a big picture of climate risk at the global level is critical to decision-making at the international level $^{6}$ (Box 1).

The risk levels assessed in the 2018-2019 IPCC Special Reports (on $1.5^{\circ} \mathrm{C}^{7}$, land ${ }^{8}$, and the ocean and cryosphere ${ }^{9}$ ) were illustrated by the iconic burning ember diagrams $s^{6}$, that is, vertical bars coloured from white to purple to depict a relative risk scale as a function of increasing global mean surface temperature. The IPCC distinguishes among four qualitative risk levels, from 'Undetectable' (risks that are undetected), to 'Moderate' (detectable with at least medium confidence), 'High' (significant and widespread) and 'Very high' (very high probability of severe risks and significant irreversibility or persistence of impacts). The present paper builds on a novel approach that translates the Special Reports risk levels into calibrated scores, and visualizes them as 'burning circles' (see Methods section and Supplementary Information Section 3.1). This framing allows the collection of the burning embers that are to date scattered in various reports and, more importantly, their aggregation in a consistent way ${ }^{10}$. Our dataset includes seven risk scores (four risk levels and the transition between them; from 0 to 6; Methods and Supplementary Data Table 2) and a total of 43 'items' used in the Special Reports to develop risk levels and associated burning embers (Fig. 1; list in Methods and Supplementary Information Section 2). These risk items refer to natural and human systems and sectors, and comprise physical processes, species, ecosystems and their services, economic activities, non-economic sectors and human settlements. The analysis below considers subsets of items depending on the type of information provided in the Special Reports (Supplementary Information Table 1), to contrast risks at two time horizons (present-day and end of the 21st century, hereafter end-century), four climate scenarios (RCP2.6 versus RCP8.5, and $+1.5^{\circ} \mathrm{C}$ versus $+2{ }^{\circ} \mathrm{C}$ ) and two societal adaptation scenarios (low versus ambitious). Risk levels and associated confidence levels (median for composite values) as defined in the Special Reports are reported in single quotation marks (Supplementary Data Table 2).

Our scoring system does not suggest that climate risk levels can be directly quantified on an absolute scale, nor that there are straightforward implications in terms of environmental economics (for example, any correlative increase between risk scores and costs of damages). Rather, the purpose of aggregation is to advance knowledge on relative risk in three major areas: climate risk amplification across warming scenarios; the anticipated benefits of ambitious adaptation for risk reduction; and the potential for residual risks (that is, risks that remain despite adaptation ${ }^{7}$ ) and limits to adaptation at the global scale.

While offering a new level of global synthesis, this approach has three main limitations (see concluding section). First, it assumes a linear development of risk, despite acknowledging that risk could change non-linearly at century scale, that is, both exponentially and/ or with jumps. Second, systemic feedbacks between different risks are not comprehensively considered due to unknowns ${ }^{11}$ regarding the inter-connectedness of natural and human systems over many spatial scales and, consequently, to knowledge gaps on both risk amplification factors ${ }^{12}$ and the potential for positive outcomes of

'Institute for Sustainable Development and International Relations (IDDRI-Sciences Po), Paris, France. '2LIENSs laboratory UMR7266, La Rochelle University-CNRS, La Rochelle, France. ${ }^{3}$ Alfred Wegener Institute, Bremerhaven, Germany. ${ }^{4}$ Department of Geography, Ludwig-Maximilians-Universität München (LMU), München, Germany. ${ }^{5}$ Instituto Argentino de Oceanografía (IADO), Consejo Nacional de Investigaciones Científicas y Técnicas (CONICET), Bahía Blanca, Argentina. ${ }^{6}$ United Nations Office for Disaster Risk Reduction, Bonn, Germany. ${ }^{7}$ ARC Centre for Excellence in Coral Reef Studies, School of Biological Sciences, University of Queensland, Queensland, St Lucia, Australia. ${ }^{8}$ Sorbonne University, CNRS, Laboratoire d'Océanographie de Villefranche, Villefranche-sur-mer, France. ${ }^{9}$ Ocean Acidification and other Changes: Impacts and Solutions Initiative, Prince Albert II of Monaco Foundation, Monaco, Principality of Monaco. ${ }^{10}$ Present address: United Nations Office for the Coordination of Humanitarian Affairs, United Nations Headquaters, New York, NY, USA. 凶e-mail: alexandre.magnan@iddri.org 
Besides technical issues, an important and recurring question raised by global composite risk metrics relates to their usefulness. The development of this paper has been partly inspired by the discussions held within the Structured Expert Dialogue (SED) of the UNFCCC in November 2020, in the follow-up of the release of the three IPCC Special Reports on which the present study is based. SED brings together IPCC authors and national representatives in climate negotiations to exchange views, information and ideas in an open and transparent manner. As stated by the co-facilitators of the November 2020 SED, the exercise especially aims at "enhancing Parties' understanding of the long-term global goal (LTGG) and scenarios towards achieving it, progress made in relation to addressing information and knowledge gaps, and challenges and opportunities; and assessing the overall aggregated effect of the steps taken by Parties to achieve the LTGG" (https://unfccc.int/sites/ default/files/resource/SED_2020_InfoNote.pdf; p. 3). An important outcome for these reflections is the first UNFCCC Global Stocktake in 2023 that will assess the progress made on global mitigation and adaptation. While major efforts to analyse aggregated efforts on mitigation have been undertaken and are regularly revised, aggregated global-scale information on the impacts and risks associated with mitigation scenarios and warming levels is still lacking. The present paper combines the risk analyses developed in the three IPCC Special Reports to provide an overview of composite risk of anthropogenic climate change at the global level. The added value of such a big picture of global climate risk is twofold:

(1) It can clarify the challenges of both mitigation (that is, how much climate risk could be avoided globally through enhanced mitigation) and adaptation (that is, to what extent could ambitious adaptation efforts reduce risk levels globally and what are the associated levels of residual risks). This is critical information to stimulate more ambitious policy targets and nationally determined contributions, and galvanize climate action at national levels across a wide diversity of systems (natural and human) and regions (ocean, coasts, land).

(2) It emphasizes that climate adaptation is not only a national or local issue but also a global concern, thereby offering a metric that may contribute to a more practical understanding of the
Global Goal on Adaptation established at the 21st Conference of the Parties to the UNFCCC in 2015.

The approach used in this paper to set up a composite value of climate risk globally could contribute to the LTGG in the perspective of the Global Stocktakes (in 2023 and beyond) by:

- Moving climate targets from temperature only to risk reduction levels, henceforth providing the opportunity to consider global mitigation and global adaptation goals together. Decision-makers at the international and national levels need more than temperature targets alone, but also insights on what these targets mean for ensuring climate-related human security;

- Informing the question: are we on track for climate risk reduction globally? This would add to the policy landscape by refining the Global Goal on Adaptation, the definition of which-enhancing adaptive capacity, strengthening resilience and with a view to contributing to sustainable developmentis broad and imprecise, and leads to very subjective interpretations of adaptation targets ${ }^{37}$. We argue that a more specific framing using a composite index for future global climate risk based on expert judgements addressing multiple ecological and human systems across latitudes and development levels, would facilitate the identification of critical areas of risk across the globe, and therefore 'shared adaptation goals' here defined as a more disaggregated understanding of the Global Goal on Adaptation;

- Improving the communication of climate risk by the IPCC through a cross-system understanding of risk levels under various global warming scenarios (the 'big picture') and the move from individual burning embers to burning circles combining all risk items considered in the Special and Main Reports of a given IPCC cycle. This would be of added value especially for the Summary for Policy Makers of Working Group II (Impacts, Vulnerability, Adaptation) and the cross-Working Group Synthesis Report. This could be critical information to facilitate future dialogue with the policy community (for example, through Structured Expert Dialogues). climate change that would lower risk levels. Third, because different communities or sectors value risks differently, it remains challenging to define a universal risk metric and risk levels that could be considered 'tolerable" ${ }^{13}$ globally. On the other hand, a composite metric can be useful to answer recurrent questions about the big picture' of risk induced by anthropogenic climate change globally, that is, when all systems are considered simultaneously (Box 1). Such view has been foundational to the development of this paper and justifies the use of a numerical descriptor to bring all risks together. As with any synthesis work communicating conclusions from a body of evidence instead of the evidence itself, it however generates some 'uncertainty absorption', that is, when some distance from the original data limits the ability of the recipients to judge the correctness of the information ${ }^{14}$. To address this concern, we: (1) assigned equal weights to individual risk scores to avoid any value judgement on the relative importance of a wide range of risks; (2) developed a sensitivity analysis to ascertain that high-level conclusions are robust over a wide range of weighting assumptions (results from the equal-weighting approach are reported in the text; see Methods and Supplementary Information Sections 3.3 to 3.6); and (3) systematically associated qualitative and quantitative statements to increase the consistency of interpretation among recipients ${ }^{15}$.

\section{Observed multi-dimensional global climate risk}

Evidence for climate change-related impacts and risks is already widespread globally ${ }^{16-19}$, affecting not only the land and ocean surfaces (horizontal dimension), but also the deep ocean (vertical dimension). Observed impacts are reported for 34 out of the 43 items studied (mostly with 'Medium' to 'High confidence'; Supplementary Data Table 3), and at least at a 'Moderate' level of risk for 22 items. Warm-water coral reefs are the only exception as 'High' to 'High-to-very high' risk is reported at the present day with 'Very high confidence ${ }^{\prime 6,17,19}$. At the other end of the risk spectrum, current risk remains difficult to detect and attribute to anthropogenic climate change for deep sea ecosystems ${ }^{20}$ (Abyssal plains, Cold-water corals, Seamounts-canyons-slopes, as well as Hydrothermal vents and methane seeps), for mid-latitude Bivalves and bivalve fisheries and aquaculture, Mangrove forests, Eastern boundary upwelling systems and Estuarine ecosystems ${ }^{16}$, as well as for Salt marshes, Sandy beaches and Rocky shores ${ }^{9}$.

\section{Projected global climate risk}

The IPCC Special Reports allow contrasting global composite risks under two Representative Concentration Pathways (RCPs), and for $+1.5^{\circ} \mathrm{C}$ versus $+2^{\circ} \mathrm{C}$. 


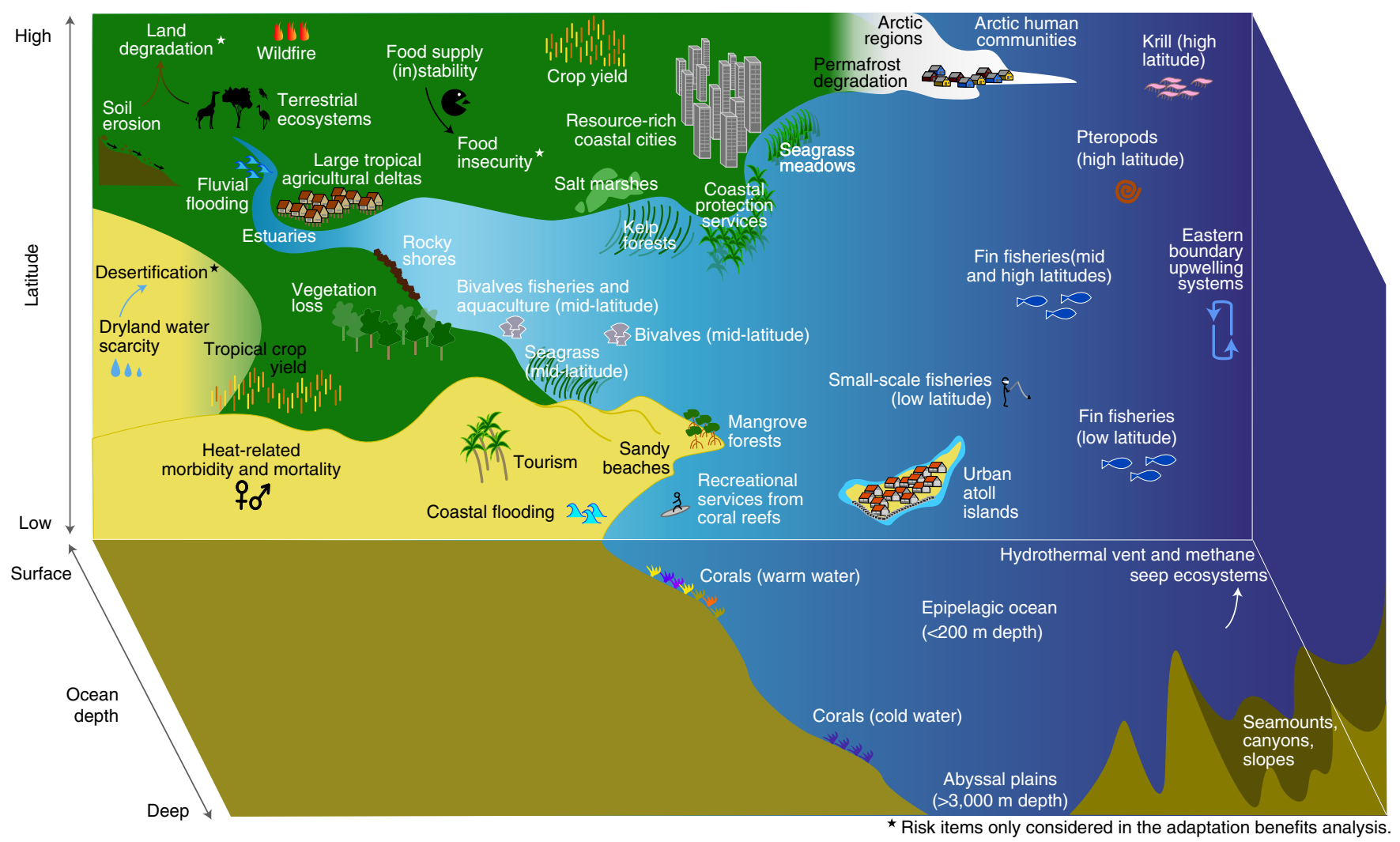

Fig. 1 | The 43 items for which the 2018 and 2019 IPCC Special Reports assessed risk levels under global warming and sea-level rise. These risk items refer to physical processes, species, ecosystems, economic activities, non-economic sectors and human settlements. Each of them is defined in Supplementary Information Sections 2.1 and 2.2.

Contrasting RCPs. Refer to Fig. 2, Supplementary Information Sections 3.1 and 3.3, and Supplementary Table 4a-c. The Special Reports assess risk in relation to global mean surface temperature (GMST), and corresponding sea surface temperature (GMSST) and sea-level rise (GMSLR). Together, they provide information against RCP2.6 and RCP8.5 for 25 items covering both natural and human systems (Supplementary Information Table 1). Considering this subset, the analysis shows that compared to present-day risk level, global climate risk will rise substantially by the end of the century under RCP2.6 and RCP8.5, the composite score being multiplied by a factor of $\sim 2$ and $\sim 4$, respectively. This result is assigned a 'Medium confidence' level (median), which characterizes seven IPCC estimates out of ten for both RCPs; no risk level is associated with a 'Very high confidence' level, or even a 'High confidence' level under RCP8.5.

Mean global climate risk is 'Moderate' and 'High-to-very high' under RCP2.6 and RCP8.5, respectively. While only 4 out of the 25 items exhibit at least 'High' risk by the end of the 21 st century under RCP2.6 (for example, Food supply instability or risk from Permafrost degradation), this number increases to 8 under RCP8.5, and includes Abyssal plains, Sandy beaches, Arctic human communities and ecological risks from Soil erosion.

Under RCP2.6, the increase in present-day mean risk for natural and human systems categories as a whole is close to the total mean (that is, $\times 1.8$; Supplementary Information Table $4 \mathrm{~b}$ ), but is higher for ecological risk from Vegetation loss, economic and livelihood risk from Dryland water scarcity, and risk to infrastructure and human health from Wildfire. The gap between natural and human systems widens under RCP8.5: the increase in the mean risk for natural systems is higher than the total mean $(\times 4.5$ compared with $\times 3.9)$, while being more modest for human systems $(\times 3.0)$. For 10 out of the 17 items of the natural systems considered here, 'Undetectable' to 'Undetectable-to-moderate' risk level today will increase under RCP8.5 to 'High' (adding Mangrove forests, Estuaries, and ecosystems in Abyssal plains and Seamounts-canyons-slopes), 'High-to-very high' (Eastern boundary upwelling systems) or 'Very high' (Cold-water corals, Rocky shores, Vegetation loss).

Overall, the results show a substantial amplification of the end-century global climate risk from RCP2.6 to RCP8.5 ( $\times 2.2$ of the composite risk score; Supplementary Information Table $4 \mathrm{~b})$. The RCP2.6 to RCP8.5 amplification is lower for human systems than for natural systems $(\times 1.6$ and $\times 2.5$, respectively). This may be partly due to a lag in the propagation of risk from climate-related changes in physical and chemical variables (temperature, $\mathrm{pH}$, sea level, etc.) to impacts on ecosystems and services, and finally to human assets and activities ${ }^{21}$. While human systems can be affected directly by physical changes, for example, through sea-level changes damaging built assets ${ }^{18}$, a substantial number of risks develop through impacts on natural systems, including ecosystem services and the availability of resources (for example, freshwater, soils, etc.). This is particularly the case for the human systems considered in this study, which are all closely dependent on environmental conditions, for example, risk of economic loss and declines in livelihoods from water scarcity on drylands (Dryland water scarcity), or risk to coastal communities living in climate-sensitive environments affected by sea-level rise and ocean changes (for example, Arctic human communities, Urban atoll islands). Therefore, because they are 'located' downstream in the risk cascade, human systems may experience a lag in terms of the full consequences of climate change. Importantly, this hypothesis implies that end-century risks to human systems will 

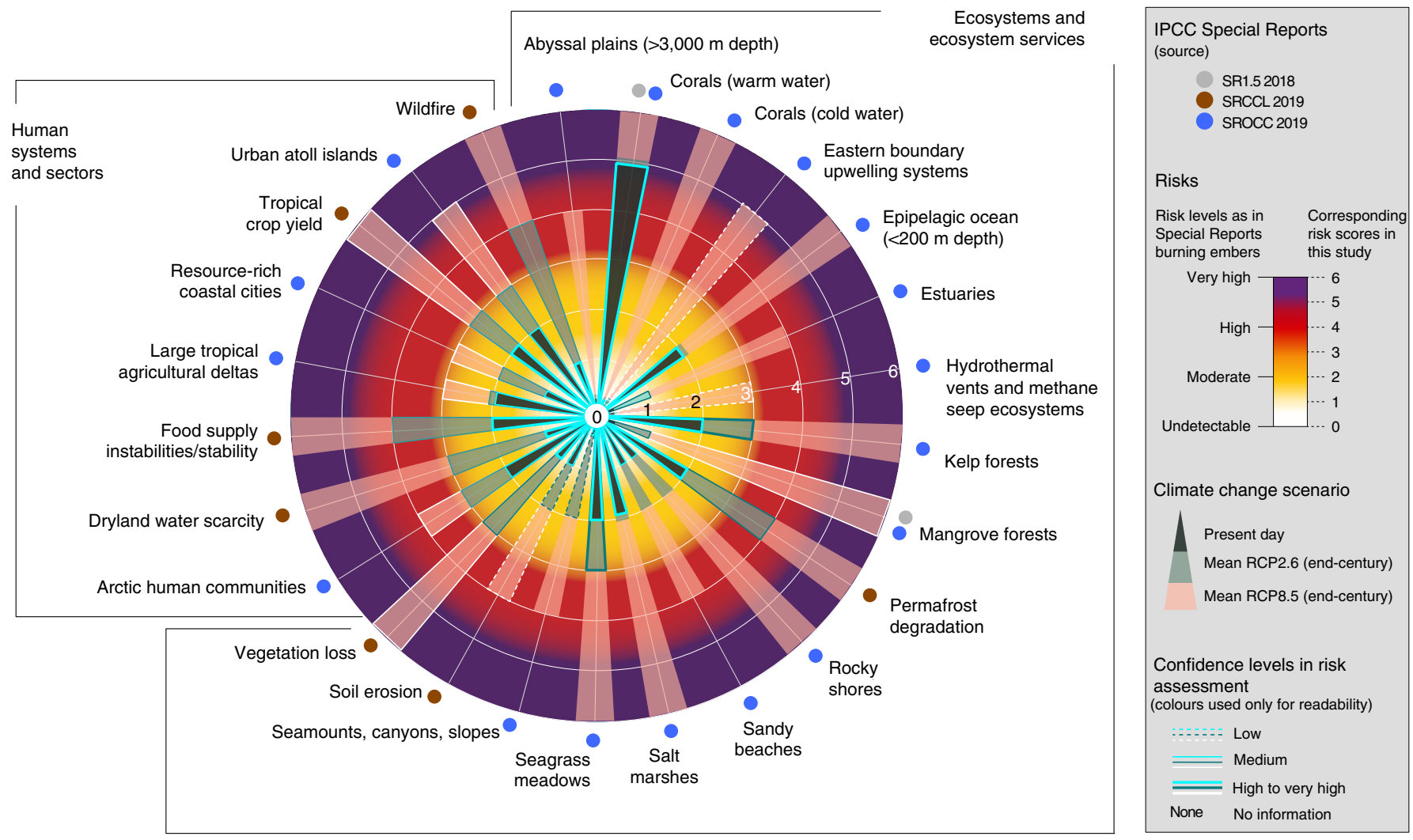

Fig. 2 | Global climate risk under contrasting RCPs by the end of the 21st century. Global warming levels considered are $\sim 1.6^{\circ} \mathrm{C}$ for mean RCP2.6 and $\sim 4.3^{\circ} \mathrm{C}$ for mean RCP8.5 by the end of the $21 \mathrm{st}$ century relative to pre-industrial $(1850-1900)^{35,36}$ levels. Sea-level rise considered is $+43 \mathrm{~cm}$ and $+84 \mathrm{~cm}$ compared to present day (1986-2005). SR1.5, IPCC Special Report on Global Warming of 1.5 ${ }^{\circ}$; SRCCL, IPCC Special Report on Climate Change and Land; SROCC, IPCC Special Report on Ocean and Cryosphere in a Changing Climate. For source data, see Supplementary Information Section 3.3 and Supplementary Data Table 4a.

keep rising after the end of this century or once a given warming level is exceeded (see next subsection), especially in the context of long-term commitment to climate change ${ }^{7,18}$.

$1.5^{\circ} \mathrm{C}$ versus $2^{\circ} \mathrm{C}$. Refer to Fig. 3, Supplementary Information Sections 3.1 and 3.4, and Supplementary Table 5a-c. Considering the subset of 33 items for which information on risks under $+1.5^{\circ} \mathrm{C}$ is available in the Special Reports ${ }^{16,17,19}$, the analysis shows that mean global climate risk rises from 'Undetectable-to-moderate' at present day to 'Moderate-to-high' under both $1.5^{\circ} \mathrm{C}$ and $2{ }^{\circ} \mathrm{C}$ global warming above pre-industrial level. This result is assigned a 'Medium confidence' level, which characterizes five IPCC estimates out of ten for both $+1.5^{\circ} \mathrm{C}$ and $+2{ }^{\circ} \mathrm{C}$, with another six being assigned a 'High confidence' level. Under both RCPs, only risk levels to Warm-water corals are associated with a 'Very high confidence' level.

Mean risk scores for natural and human systems are projected to experience a similar increase from the current $\sim 0.9^{\circ} \mathrm{C}$ of global warming above pre-industrial level to $1.5^{\circ} \mathrm{C}(\times 2.0$ and $\times 1.8$, respectively), as well as to $+2{ }^{\circ} \mathrm{C}(\times 2.8$ and $\times 2.3$, respectively). The increase in risk scores from $1.5^{\circ} \mathrm{C}$ to $2^{\circ} \mathrm{C}$ is also similar for natural and human systems $(\times 1.4$ and $\times 1.2$, respectively), suggesting a substantial increase in mean global climate risk from $+1.5^{\circ} \mathrm{C}$ to $+2{ }^{\circ} \mathrm{C}$ (by about $\sim 30 \%$ for combined natural and human systems; Supplementary Information Table $5 b$ ). However, such increase will not be uniform across the risk items. Six items will reach the 'High' risk level threshold from $1.5^{\circ} \mathrm{C}$ to $2^{\circ} \mathrm{C}$ of warming, and five out of the nine items showing at least 'High' risk under $1.5^{\circ} \mathrm{C}$ warming will experience higher risk with an additional $0.5^{\circ} \mathrm{C}$ of global warming. The risk score approximately doubles from $+1.5^{\circ} \mathrm{C}$ to $+2^{\circ} \mathrm{C}$ for Coastal protection services, Rocky shores, Salt marshes and Terrestrial ecosystems ${ }^{16-19}$, as well as for mid-latitude Bivalve fisheries and aquaculture ${ }^{19}$. At the other end of the risk spectrum, the $+1.5^{\circ} \mathrm{C}$ risk score is projected to remain stable with a $0.5^{\circ} \mathrm{C}$ increase in GMST for some marine ecosystems and habitats (Epipelagic ecosystems, Mangrove forests, Sandy beaches and Seagrass meadows), as well as for ecological risk from Soil erosion. This is also the case for more than half of the human systems considered, especially Fin fisheries at low and mid/high latitudes, Heat-related mortality and morbidity, Recreational services from coral reefs, and low-latitude Small-scale fisheries. Warm-water coral reefs continue to experience 'Very high' risk at $+1.5^{\circ} \mathrm{C}$ of global warming ${ }^{16-19}$.

These estimates do not consider the rate of warming, that is how fast a given temperature level will be reached or exceeded, and the implications on species and socio-ecological systems. It can be hypothesized $^{6}$ that higher rates of warming will lead to earlier approach of risk thresholds and to accelerating cascading consequences within each risk item, which would result in higher increments in risk levels.

Finally, the fact that the amplifying factor of the mean risk score is slightly lower for human systems compared with natural systems under all warming scenarios reinforces the hypothesis of a lag in the propagation of risk. 


\section{Risk under a $1.5^{\circ} \mathrm{C}$ and $2{ }^{\circ} \mathrm{C}$ global warming, end of the 21 st century}
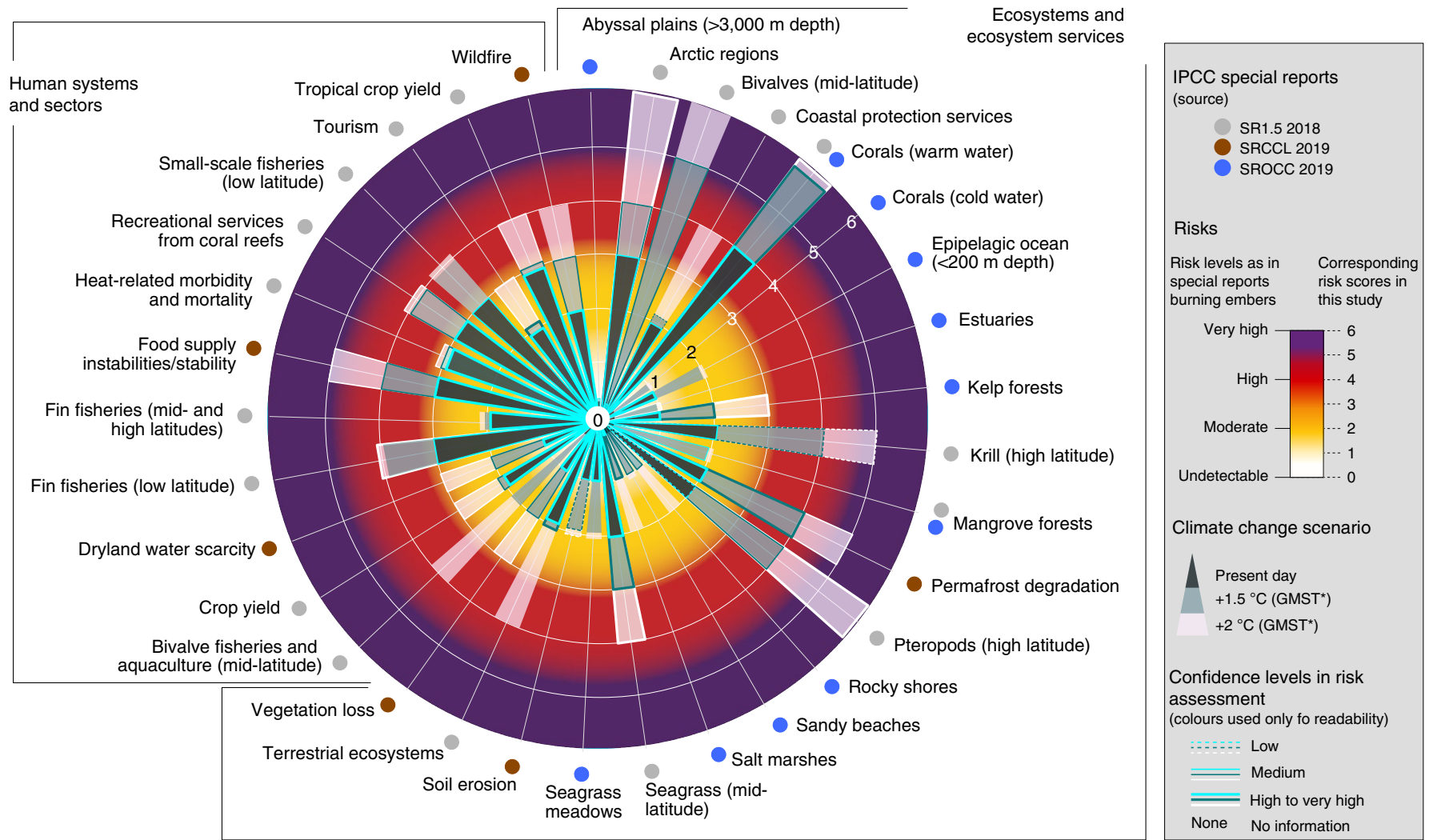

Fig. 3 | Global climate risk under a $1.5^{\circ} \mathrm{C}$ and $2^{\circ} \mathrm{C}$ global warming. Present day refers to the $2006-2015$ period, and global warming levels are relative to pre-industrial (1850-1900) levels. For source data, see Supplementary Information Section 3.4 and Supplementary Data Table 5a.

Regional disparities. Refer to Supplementary Information Section 3.6 and Supplementary Table $7 \mathrm{a}-\mathrm{c}$. A first comparison among Ocean, Coast and Land environments (Supplementary Information Table 1) suggests a potential higher sensitivity to anthropogenic climate change of the Ocean subset of risk items compared with the Coast and Land subsets, especially when moving from RCP2.6 to RCP8.5-Ocean risk amplification is double that of Coast and Land. In absolute terms, however, the end-century median risk levels are higher for Coast and Land subsets under both warming scenarios, reaching for example, 'Very high' risk under RCP8.5 compared with 'High' risk (median) for the Ocean subset. By contrast, the three subsets show similar patterns from $1.5^{\circ} \mathrm{C}$ to $2^{\circ} \mathrm{C}$ of warming, with risk reaching 'Moderate-to-high' (Land) and 'High' (Ocean, Coast) median levels under $+2{ }^{\circ} \mathrm{C}$, and substantial risk amplification from $+1.5^{\circ} \mathrm{C}$ to $+2^{\circ} \mathrm{C}$ (by $\sim 30 \%$ ).

\section{Societal adaptation benefits and residual risks}

The Special Reports facilitate an exploratory assessment of the potential benefits of societal adaptation for risk reduction, as well as residual risks (that is, risks that remain despite adaptation ${ }^{7}$ ) by the end of the 21st century (Fig. 4, Supplementary Information Sections 2.1, 2.2 and 3.5, and Supplementary Table 6a-c). The Ocean and Cryosphere Special Report explicitly contrasts 'no-to-moderate' and 'maximum potential' adaptation scenarios for GMSLR risks ${ }^{9,18}$. The former describes the continuation of current adaptation efforts, whereas the latter 'represents a combination of responses implemented to their full extent and thus significant additional efforts compared to today, assuming minimal financial, social and political barriers' (ref. ${ }^{9}$, p. 34). The Land Special Report adopts a more implicit approach through the use of two Shared Socio-economic
Pathways (SSPs), SSP1 'Sustainability' and SSP3 'Regional Rivalry', that are based on societal conditions related to trends in demographics, economics, governance, etc. Although they do not consider special climate policy, the SSPs are hypothesized to make adaptation and mitigation easier or harder ${ }^{7,19,22,23}$. Together, the 'no-to-moderate' SSP3 and 'maximum potential' SSP1 configurations describe low and ambitious adaptation scenarios.

The seven items for which the Special Reports provide information on risk levels influenced by adaptation are Arctic human communities, Desertification, Food insecurity, Land degradation, Large tropical agricultural deltas, Resource-rich coastal cities and Urban atoll island $s^{8,9,17,18}$ (Supplementary Information Table 1). Ambitious societal adaptation is projected to reduce present-day global climate risk by $\sim 40 \%$ by the end of this century under both RCP2.6 (decrease in mean risk level from 'Moderate-to-high' to 'Moderate') and RCP8.5 (from 'High-to-very high' to 'Moderate-to-high'). This result has a 'Medium confidence' level under RCP2.6 and a 'Low confidence' level under RCP8.5, requiring caution in any generalization. Furthermore, under both RCP2.6 and RCP 8.5 and both low and ambitious adaptation scenarios, none of the estimates is associated with a 'High' or 'Very high confidence' level.

Keeping this in mind, such a finding suggests that even ambitious adaptation efforts globally do not have the potential to offset the substantial increase in risk associated with each additional $0.5^{\circ} \mathrm{C}$ of global warming $\left(\sim 30 \%\right.$ increase in risk score; see the $+1.5^{\circ} \mathrm{C}$ versus $+2{ }^{\circ} \mathrm{C}$ analysis above). It is likely that this estimate cannot be linearly interpolated between RCP2.6 and RCP8.5 (that is, $\sim 3^{\circ} \mathrm{C}$ difference in GMST) but rather exponentially (see discussion below). Substantial residual risks will therefore remain despite very active 

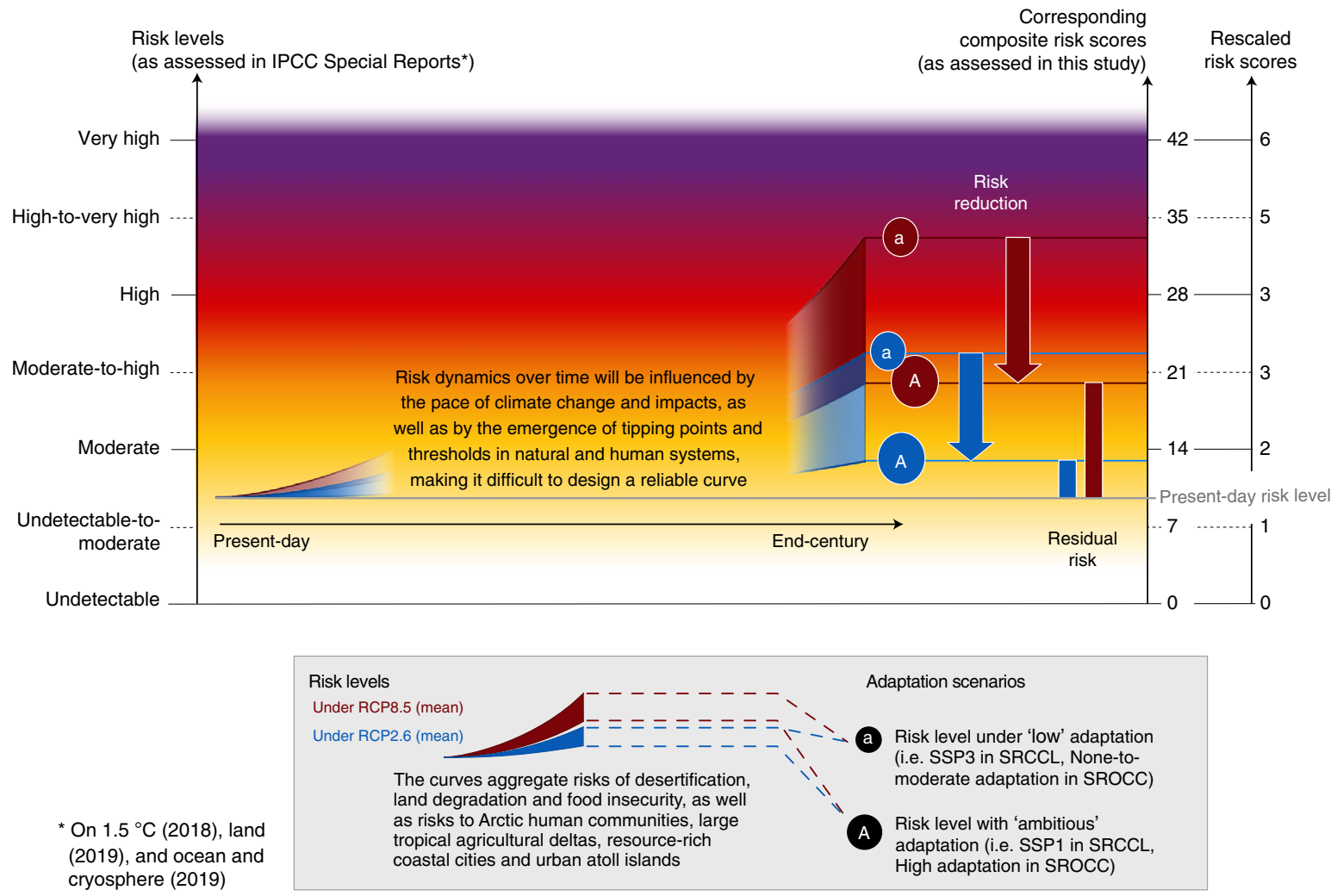

Fig. 4 | Societal adaptation benefits and residual risks by the end of the 21st century. The figure shows global climate risk increase for an aggregation of seven risk items assessed in IPCC recent Special Reports $8,9,1,1,18$. It considers the present-day and the end-century levels under both RCP2.6 and RCP8.5, and two adaptation scenarios (low and ambitious, ' $a$ ' and 'A' bubbles, respectively; for details, see the main text and Supplementary Information Section 3.5). Vertical arrows and bars represent composite risk score reduction through adaptation and residual risks, respectively, under RCP2.6 (blue) and RCP8.5 (brown). For source data, see Supplementary Information Section 3.5 and Supplementary Data Table 6a.

mitigation and adaptation globally (Supplementary Data Table 6a and Fig. 4). While global climate residual risk by the end-century could be minimized under RCP2.6 to an approximately one-third increase in present-day mean risk score, it is expected to represent a doubling of today's mean risk score under RCP8.5.

\section{Discussion}

The study shows that by the end of the 21 st century, even a low greenhouse gas emission trajectory will lead to an important increase in today's global climate risk level (doubling of composite risk score), and to an even more substantial increase (fourfold) under a high emission pathway. It is anticipated, for example, that about a quarter of the risk items considered in this study will experience 'High' to 'Very high' risks by the end of this century even under RCP2.6, and that 16 out of 25 items will reach or exceed the 'High' risk threshold under RCP8.5. The study also shows that societal adaptation has the potential to decrease the end-century global climate risk by a significant amount $(\sim 40 \%)$ under all emission scenarios, but cannot entirely eliminate future risks from anthropogenic climate change. Such conclusions call for a dramatic scaling up of global mitigation and societal adaptation together. Interestingly, the fact that climate change will disproportionately affect less-developed countries ${ }^{7,8}$ as well as the poorest segments of the population, also in industrialized countries ${ }^{24}$, emphasizes the need for climate action to be implemented in the context of more equitable environmental and social development pathways, which strongly resonates with similar requirements identified for the Covid-19 pandemic and therefore highlights synergies across global policy issues ${ }^{25}$.
This study also reveals three important scientific challenges that need to be addressed globally: evaluating the escalating and systemic nature of climate change-related risk, assessing the effectiveness of adaptation, and understanding limits to adaptation and residual risks. Addressing these challenges would substantially advance knowledge on global climate risk, with multiple expected benefits (Box 1).

Evaluating the escalating and systemic nature of climate risk globally. As mentioned earlier and illustrated in Fig. 4, this study underestimates the possible non-linear nature of risk development over time, as well as the influence of interactions among the risk items considered (Fig. 1), that is, systemic risk. It acknowledges, for example, that interactive effects between climatic and non-climatic drivers such as land use, pollution and overfishing, lead to substantial regional variability in the levels of exposure and vulnerability $^{26}$, adding complexity to the global risk assessment ${ }^{12}$. Advancing knowledge on risk propagation calls for exploring two scientific frontiers. First, a better understanding of the major risk thresholds in the climate, natural and human systems ${ }^{27,28}$ is needed, including which global warming levels they are associated with, when they could occur (given various rates of global warming), and how they could affect risk development. The IPCC Special Reports suggest some of these thresholds, for example, at $1.5^{\circ} \mathrm{C}$ of global warming for Warm-water coral reefs ${ }^{16-19}$ ('Very High' risk level) and Food security ${ }^{17}$ and various types of fisheries ${ }^{16}$ ('High' risk levels) (Fig. 3). A more comprehensive approach should consider cascading effects and tipping points for a broader range of systems and geographies ${ }^{12}$. Second, although compounding risks are increasingly recognized at 
all scales ${ }^{29-31}$, no agreed-upon risk amplification factor has emerged in the climate change literature that could help assess the extent to which the global climate risk is more than the sum of individual risks. This aligns with previous calls for a 'Global System Science' 11 to emerge.

Assessing the effectiveness of societal adaptation globally. The above analysis of the potential for ambitious adaptation to reduce climate risk relies on a limited set of data (seven items) and different assumptions, that is, low versus high adaptation scenarios on the one hand, and contrasting SSPs on the other hand. Advancing knowledge requires a more consistent approach, for example, through the development of shared adaptation scenarios across risk items and reports, and a wider range of assessments considering future risk levels with and without adaptation.

Understanding limits to societal adaptation globally. The finding that ambitious adaptation cannot fully control the continuous increase in residual risks when mitigation fails (Fig. 4, right), suggests the existence of hard adaptation limits at the global scale. In contrast to 'soft' limits for which options to avoid intolerable risks may exist but are currently not available, hard limits cannot be overcome $^{32}$. Adaptation limits are usually considered context-specific as they depend on local physical, environmental and societal circumstances; here we hypothesized that they also need to be considered as a global concern. The potential continuous increase in residual risks across systems indeed raises the issue of risk accumulation over time and associated increasing inherited burden in terms of adaptation needs, therefore highlighting intergenerational equity concern $s^{33}$. More research is needed to better characterize the time component of adaptation limits at the global scale (when they will occur), depending on their nature (physical, socio-economic, institutional; hard versus soft) as well as on the rate of impacts, and possibly identify ecological, geographical and societal hotspots. This could represent critical information for the ongoing Loss and Damage mechanism under the UNFCCC ${ }^{34}$.

\section{Online content}

Any methods, additional references, Nature Research reporting summaries, source data, extended data, supplementary information, acknowledgements, peer review information; details of author contributions and competing interests; and statements of data and code availability are available at https://doi.org/10.1038/ s41558-021-01156-w.

Received: 15 December 2020; Accepted: 18 August 2021; Published online: 30 September 2021

\section{References}

1. Rockstrom, J. et al. A safe operating space for humanity. Nature 461, 472-475 (2009).

2. Steffen, W. et al. Planetary boundaries: guiding human development on a changing planet. Science 347, 1259855 (2015).

3. Nash, K. L. et al. Planetary boundaries for a blue planet. Nat. Ecol. Evol. 1, 1625-1634 (2017)

4. Lade, S. J. et al. Human impacts on planetary boundaries amplified by Earth system interactions. Nat. Sustain. 3, 119-128 (2020).

5. Smith, J. et al. in Climate Change 2001: Impacts, Adaptation, and Vulnerability (eds McCarthy, J. J. et al.) 913-967 (IPCC, Cambridge Univ. Press, 2001).

6. Zommers, Z. et al. Burning embers: towards more transparent and robust climate change risk assessments. Nat. Rev. Earth Environ. 1, 516-529 (2020).
7. IPCC Special Report on Global Warming of $1.5^{\circ} \mathrm{C}$ (eds Masson-Delmotte, V. et al.) (WMO, 2018)

8. IPCC Special Report on Climate Change and Land (eds Shukla, P. R. et al.) (WMO, 2019).

9. IPCC Special Report on Ocean and Cryosphere in a Changing Climate (eds Pörtner, H.-O. et al.) (WMO, 2019).

10. Magnan, A. K. et al. Implications of the Paris Agreement for the ocean. Nat. Clim. Change 16, 732-734 (2016).

11. Helbing, D. Globally networked risks and how to respond. Nature 497, 51-59 (2013).

12. Simpson, N. P. et al. A framework for complex climate change risk assessment. One Earth 4, 489-501 (2021).

13. Handmer, J. \& Nalau J. in Loss and Damage from Climate Change (eds Mechler, R. et al.) 365-380 (Springer, 2018).

14. March, J. G. \& Simon, H. A. Organizations 2nd edn (Wiley-Blackwell, 1993)

15. Budescu, D. V., Por, H.-H. \& Broomell, S. B. Effective communication of uncertainty in the IPCC reports. Clim. Change 113, 181-200 (2012).

16. Bindoff, N. L. et al. in IPCC Special Report on Ocean and Cryosphere in a Changing Climate (eds Pörtner, H.-O. et al.) 447-587 (WMO, 2019).

17. Hurlbert, M. et al. in IPCC Special Report on Climate Change and Land (eds Shukla P. R. et al.) 673-800 (WMO, 2019).

18. Oppenheimer, M. et al. in IPCC Special Report on Ocean and Cryosphere in a Changing Climate (eds Pörtner H.-O. et al.) 321-445 (WMO, 2019).

19. Hoegh-Guldberg, O. et al. in IPCC Special Report on Global Warming of $1.5^{\circ} \mathrm{C}$ (eds Masson-Delmotte V. et al.) 175-311 (WMO, 2018).

20. Brito-Morales, I. et al. Climate velocity reveals increasing exposure of deep-ocean biodiversity to future warming. Nat. Clim. Change 10, 576-581 (2020)

21. Gattuso, J.-P. et al. Contrasting futures for ocean and society from different anthropogenic $\mathrm{CO}_{2}$ emissions scenarios. Science 349, aac4722 (2015).

22. Jones, B. \& O'Neill, B. Spatially explicit global population scenarios consistent with the Shared Socioeconomic Pathways. Environ. Res. Lett. 11, 084003 (2016).

23. Andrijevic, M., Cuaresma, J. C., Muttarak, R. \& Schleussner, C.-F. Governance in socioeconomic pathways and its role for future adaptive capacity. Nat. Sustain. 3, 35-41 (2020).

24. Pelling, M. \& Garschagen, M. Put equity first in climate adaptation. Nature 569, 327-329 (2019)

25. Hepburn, C., O’Callaghan, B., Stern, N., Stiglitz, J. \& Zenghelis, D. Will COVID-19 fiscal recovery packages accelerate or retard progress on climate change? Oxf. Rev. Econ. Policy https://doi.org/10.1093/oxrep/graa015 (2020)

26. Williamson, P. \& Guinder, V. A. in The Impacts of Climate Change: A Comprehensive Study of Physical, Biophysical, Social and Political Issues (ed. Letcher, T. M.) Ch. 4 (Elsevier, 2021).

27. Steffen, W. et al. Trajectories of the Earth system in the Anthropocene. Proc. Natl Acad. Sci. USA 115, 8252-8259 (2018).

28. Lenton, T. M. et al. Climate tipping points-too risky to bet against. Nature 575, 592-595 (2019)

29. Zscheischeler, J. et al. Future climate risk from compound events. Nat. Clim. Change 8, 469-477 (2018).

30. Zscheischeler, J. et al. A typology of compound weather and climate events. Nat. Rev. Earth Environ. 1, 333-347 (2020).

31. The Global Risks Report 2020 15th edn (WEF, 2020); http://www3.weforum org/docs/WEF_Global_Risk_Report_2020.pdf

32. Weyer, N. M. et al. in IPCC Special Report on Ocean and Cryosphere in a Changing Climate (eds Pörtner, H.-O. et al.) 677-702 (WMO, 2019).

33. Weiss, E. B. Climate change, intergenerational equity and international law: an introductory note. Clim. Change 15, 327-335 (1989).

34. Mechler, R. et al. (eds) Loss and Damages from Climate Change (Springer, 2018); https://doi.org/10.1007/978-3-319-72026-5 1

35. Collins, M. et al. in Climate Change 2013: The Physical Science Basis (eds Stocker T. F. et al.) 2019-1136 (IPCC, Cambridge Univ. Press, 2013).

36. Allen, M. R. et al. in IPCC Special Report on Global Warming of $1.5^{\circ} \mathrm{C}$ (eds Masson-Delmotte, V. et al.) 49-91 (WMO, 2018).

37. Magnan, A. K. \& Ribera, T. Global adaptation after Paris. Science $\mathbf{3 5 2}$, 1280-1282 (2016).

Publisher's note Springer Nature remains neutral with regard to jurisdictional claims in published maps and institutional affiliations.

(c) The Author(s), under exclusive licence to Springer Nature Limited 2021 


\section{Methods}

The Supplementary Information provides more details and includes the full database (Supplementary Data); a glossary of the 43 items studied (Supplementary Information Section 2); and a description of the general method and specific investigations on contrasting RCPs, $1.5^{\circ} \mathrm{C}$ versus $2^{\circ} \mathrm{C}$, regional disparity and adaptation benefits (Supplementary Information Section 3 ).

Overall approach. The goal of this paper is to use the 43 risk items analysed in three IPCC Special Reports ${ }^{7-9,16-19}$ to gain an understanding of the global risk induced by anthropogenic climate change, that is, when all risk items are combined. While the qualitative IPCC risk language describing risk levels ('Undetectable', 'Moderate,' 'High', 'Very high') allows for some intuitive visualization of risk levels under various warming scenarios (that is, the burning embers; see Box 1), the use of a quantitative risk scale helps in better understanding the location of a given risk on the risk spectrum, and therefore what a given risk increase represents. The qualitative IPCC risk scale is converted to a quantitative scale (from 0 to 6 ; see below)

Material collection. The risk assessments which led to the production of burning ember diagrams in the three IPCC Special Reports were collated. The Special Reports chapters and associated Supplementary Information have been used as the primary material. Taken together, they provide details on both the scope of the risks considered for various 'items' referring to natural $(\mathrm{N})$ and human $(\mathrm{H})$ systems (physical processes, species, ecosystems and their services, economic activities, non-economic sectors and human settlements), as well as on risk level transitions according to either temperature thresholds $\left(1.5^{\circ} \mathrm{C}\right.$ Special Report Chapter 3 and Summary for Policy Makers ${ }^{7,19}$, Land Special Report Chapter 7 and Summary for Policy Makers ${ }^{8,17}$, Ocean and Cryosphere Special Report Chapter 5 (ref. $\left.{ }^{16}\right)$ ) or sealevel rise levels (Ocean and Cryosphere Special Report Chapter $4\left(\right.$ ref. $\left.{ }^{18}\right)$ ). Chapter 7 of the Land Special Report and Chapter 4 of the Ocean and Cryosphere Special Report also provide material for the analysis of adaptation benefits.

Our study considers a total of 43 items for which the Special Reports assessed risk levels and developed burning embers (see Fig. 1 for an overview and Supplementary Information Section 2 for a full glossary). Eight burning ember diagrams initially developed in SR1.5 have been excluded from this analysis because they refer to hazards rather than risks ('Coastal flooding' and 'Fluvial flooding'), to some interpretation of risk outcomes rather than risk itself ('Ability to achieve sustainable development goals'), or composite risks through the five Reasons for Concern ('Unique and threatened systems', 'Extreme weather events', 'Distribution of impacts', 'Global aggregate impacts' and 'Large-scale singular events').

The risk items are described in Supplementary Information Section 2 and summarized below. Among these items, 40 are used to describe risk levels under RCP2.6 versus RCP8.5 and/or $1.5^{\circ} \mathrm{C}$ versus $2^{\circ} \mathrm{C}$, and 3 additional ones are used only to discuss adaptation benefits (indicated with ${ }^{* *}$ in the following list): Abyssal plains (>3,000 m depth) (N); Arctic human communities $(\mathrm{H})$; Arctic regions $(\mathrm{N})$; Bivalves (mid-latitudes) $(\mathrm{N})$; Bivalve fisheries and aquaculture (mid-latitude) $(\mathrm{H})$; Coastal protection services $(\mathrm{N})$; Corals (cold water) $(\mathrm{N})$; Corals (warm water) $(\mathrm{N})$; Crop yields $(\mathrm{H})$; Desertification $(\mathrm{N})^{* *}$; Dryland water scarcity $(\mathrm{H})$; Eastern boundary upwelling systems $(\mathrm{N})$; Epipelagic ecosystems (<200 m depth) (N); Estuaries (N); Fin fisheries (low latitudes) $(\mathrm{H})$; Fin fisheries (mid and high latitudes) $(\mathrm{H})$; Food insecurity $(\mathrm{H})^{* *}$; Food supply instabilities/stability $(\mathrm{H})$; Heat-related morbidity and mortality $(\mathrm{H})$; Hydrothermal vent \& methane seep ecosystems (N); Kelp forests (N); Krill (high latitudes) $(\mathrm{N})$; Land degradation $(\mathrm{N})^{* *}$; Large tropical agricultural deltas $(\mathrm{H})$; Mangrove forests (N); Permafrost degradation (N); Pteropods (high latitude) $(\mathrm{N})$; Recreational services from coral reefs $(\mathrm{H})$; Resource-rich coastal cities (H); Rocky shores (N); Salt marshes (N); Sandy beaches (N); Seagrass meadows (N); Seagrasses (mid-latitude) (N); Seamounts, canyons, slopes (N); Small-scale fisheries (low latitudes) (H); Soil erosion (N); Terrestrial ecosystems (N); Tourism $(\mathrm{H})$; Tropical crop yield $(\mathrm{H})$; Urban atoll islands $(\mathrm{H})$; Vegetation loss $(\mathrm{N})$; and Wildfire $(\mathrm{H})$

Risk levels recoding and scoring system. The IPCC risk language used in the three Special Reports was recoded as risk scores assuming a linear scale, as assumed in the original IPCC assessments, and to allow for aggregation across item-level risks. Seven risk scores are used (Supplementary Information Section 3.1b and Supplementary Data Table 2): 0 ('Undetectable'), 1 ('Undetectable-tomoderate'), 2 ('Moderate'), 3 ('Moderate-to-high'), 4 ('High'), 5 ('High-to-very high') and 6 ('Very high'). The recoding applied to the risk levels identified for each item, in at least one Special Report, for either the global warming levels considered in this study by the end of the 21 st century relative to $1850-1900$ (mean RCP2.6 $\left(\sim 1.6^{\circ} \mathrm{C}\right)$ and mean RCP8.5 $\left(\sim 4.3^{\circ} \mathrm{C}\right)$ on the one hand $\mathrm{d}^{35},+1.5^{\circ} \mathrm{C}$ and $+2^{\circ} \mathrm{C}$ on the other hand), or global mean sea-level rise scenarios ${ }^{18}$ by the end of the 21 st century relative to $1986-2005$ (mean RCP2.6 ( $+43 \mathrm{~cm}$ ) and mean RCP8.5 $(+84 \mathrm{~cm})$ ). The translation into a numerical value (that is, risk score) relied on the risk level transition details provided in the Special Report chapters Supplementary Information (and initially based on structured expert-judgement exercises ${ }^{6,16,18,19}$ ), and on considering where the reference warming levels (mean RCP2.6, mean $\mathrm{RCP} 8.5,+1.5^{\circ} \mathrm{C},+2^{\circ} \mathrm{C}$ ) are within the temperature ranges identified in the Special Reports for classifying risk between 'Undetectable' and 'Very high'. For sea-level rise-related risks, the translation into scores relies on the discrete risk levels already identified in the Ocean and Cryosphere Special Report Chapter 4 (ref. ${ }^{9}$ ). The Supplementary Data summarizes all these risk scores.

Confidence levels. In the IPCC reports, "uncertainty in the risk transitions is represented through both the width of the temperature transition and the confidence level of the transition. Narrow transitions may be more informative for policymaking. However, narrowing a transition range typically comes at the cost of reducing the confidence level associated with the transition" (ref. ${ }^{6}$, p. 9). The same approach and material (chapters and their associated Supplementary Information) as the one described above for risk levels have been used to describe confidence levels. The four confidence levels used in the IPCC reports (Low, Medium, High, Very high) have been associated with a discrete, numerical value (from 1 to 4 ; see Supplementary Information Section 3.1c and Supplementary Data Table 2). When the Special Reports propose a half-way confidence (for example, Low-Medium or Medium-High), we applied a precautionary approach consisting of only considering the lowest confidence level (for example Low instead of Low-Medium, or Medium instead of Medium-High). Full results are presented in Supplementary Tables $4 \mathrm{a}, \mathrm{b}, 5 \mathrm{a}, \mathrm{b}$ and $6 \mathrm{a}, \mathrm{b}$.

Risk scores aggregation. Recoded risk scores were aggregated to highlight a more comprehensive, cross-item understanding of climate risk that is referred to as the 'global climate risk'. A simple aggregation (addition) was used, and individual risk scores were given equal weights to avoid any value judgement on the relative importance of a wide range of risks and given that to date, the IPCC has not developed such a cross-Special Reports comparison (see subsection below on sensitivity analysis). The number of composite risk values varies according to the climate scenario framing because not all Special Reports develop an end-century risk assessment for all items under both RCP2.6 and RCP8.5, and $+1.5^{\circ} \mathrm{C}$ and $+2{ }^{\circ} \mathrm{C}$ of global warming. Out of a total of 43 items considered in our study, 25 contrasted risk scores under RCPs and by the end of the 21 st century compared with pre-industrial levels (see Supplementary Information Section 3.3 and Fig. 2); 33 items contrasted risk scores under $+1.5^{\circ} \mathrm{C}$ and $+2{ }^{\circ} \mathrm{C}$ of global warming compared with pre-industrial levels (see Supplementary Information Section 3.4 and Fig. 3); and 7 items contrasted end-century risks scores under low and ambitious societal adaptation scenarios (see main text for description, Supplementary Information Section 3.5 for summary and Fig. 4).

Sensitivity analysis. A sensitivity analysis was conducted to assess the relevance of the no-weighting compared to other weighting options. For each stream of analysis (RCPs, $1.5^{\circ} \mathrm{C}$ versus $2^{\circ} \mathrm{C}$, Adaptation benefits; also for the regional analysis, see below), 15 tests were performed using various weights (alternatives contrasting 1 versus $2,3,5,10$ and 100) and rationales (for each alternative, both a rough estimate of the potential higher importance of some risks compared with others, and two random weightings). The full results are provided in Supplementary Data Table $4 \mathrm{c}$ for the RCP analysis, $5 \mathrm{c}$ for the $1.5^{\circ} \mathrm{C}$ versus $2{ }^{\circ} \mathrm{C}$ analysis, and $6 \mathrm{c}$ for the Adaptation benefits analysis. These 15 tests show that the no-weighting option (for example, for RCPs, see lines 63 to 76 in Supplementary Data Table $4 \mathrm{c}$ ) is close to the median global score of all tests taken together, indicating that no-weighting is an acceptable approach. Additionally, in the absence of risk hierarchy in IPCC Special Reports or in other studies considering all these specific risk items together, we argue that the no-weighting approach is the most suitable to avoid introducing any value judgement or cultural bias (that is, westernized vision of risk) on the respective importance of some risks compared to others. This view aligns with the growing concern in the scientific and non-academic communities towards better reflecting the diversity of world views on the severity of climate risks.

Regional disparities. The material in the Special Reports does not allow for contrasting risks per latitude (for example, high, mid and low) or geographical regions (for example, Africa, Europe, Pacific, etc.). However, it is possible to distinguish among risk items referring to ocean, coastal and terrestrial environments. The relevant risk items have therefore been selected and sorted according to an Ocean/Coastal/Land framing, and a new analysis (including sensitivity analysis; Supplementary Information Section 3.6) undertaken as described above. It considers two of the three main streams of analysis, that is, RCPs and $1.5^{\circ} \mathrm{C}$ versus $2^{\circ} \mathrm{C}$ (subsets of 25 and 32 risk items, respectively); too little information is available to also consider the 'adaptation benefits' stream. The full results are provided in Supplementary Data Table 7a,b and synthesized in Supplementary Information Table 7c.

Figures design. Present-day and end-century risk scores and associated confidence levels under RCP2.6/RCP8.5 and $+1.5^{\circ} \mathrm{C} /+2^{\circ} \mathrm{C}$, and for each relevant item were used to design burning circles in Figs. 2 and 3, respectively. Adaptation-related composite risk scores were used to develop Fig. 4. 


\section{Data availability}

The data supporting the findings of this study are available within the paper and its Supplementary Information and Supplementary Data.

\section{Acknowledgements}

We thank our respective governments as well as the World Meteorological Organization (WMO) for support during the development of the IPCC SR1.5, SRCCL and SROCC reports; all views expressed herein are the responsibility of the authors and do not necessarily reflect their respective institutions or the United Nations. We thank the following projects and organizations for financial support: the French National Research Agency ('Investissements d'avenir programme' Grant ANR-10-LABX-14-01 and STORISK Grant ANR-15-CE03-0003) (A.K.M., V.K.E.D.); the Prince Albert II of Monaco Foundation, the Veolia Foundation, the IAEA Ocean Acidification International Coordination Centre and the French Facility for Global Environment (J.-P.G., A.K.M.); the European Union and the ANR (INSeaPTION, Grant 690462) (V.K.E.D.); the Australian Research Council Laureate Fellowship and Australian Research Council Centre for Excellence in Coral Reef Studies (O.H.-G.). Finally, we thank the authors of the latest three IPCC Special Reports (SR1.5, SRCCL and SROCC) for their work in developing the burning embers, as well as P. Marbaix for helpful discussions on the burning circle figures.

\section{Author contributions}

A.K.M. and J.-P.G. designed the study and convened the author team. All authors contributed to the assessment, the various revisions of the manuscript and addressing reviewers' comments.

\section{Competing interests}

The authors declare no competing interests.

\section{Additional information}

Supplementary information The online version contains supplementary material available at https://doi.org/10.1038/s41558-021-01156-w.

Correspondence and requests for materials should be addressed to Alexandre K. Magnan.

Peer review information Nature Climate Change thanks Robert Lempert and the other, anonymous, reviewer(s) for their contribution to the peer review of this work.

Reprints and permissions information is available at www.nature.com/reprints. 